\title{
EDITORIAL
}

\section{Assessing perfusion and function in acute myocardial infarction: how and when?}

\section{A Pierard}

Heart 2003;89:701-703

Post-ischaemic myocardial perfusion is dynamic in nature, and microvascular damage may be reversible, even in an area of initial no reflow. The window for salvage of the myocardium may therefore be greater for some patients following acute myocardial infarction

\section{A} cute thrombotic coronary artery occlusion rapidly results in severe transmural ischaemia, contractile dysfunction, and a wavefront of myocardial injury and necrosis progressing from the endocardium to the epicardium. Myocardial cell injury is already present after 20-40 minutes of ischaemia. Microvascular damage develops slightly later, 60-90 minutes after occlusion.

\section{REPERFUSION VERSUS NO REFLOW}

Immediate treatment usually restores patency of the occluded artery as a result of the infusion of a thrombolytic agent or preferably of direct percutaneous coronary intervention (PCI). Optimal angiographic recanalisation does not necessarily imply tissue reperfusion. Inadequate blood flow in the microcirculation despite recanalisation, termed "no reflow phenomenon", was first studied experimentally ${ }^{2}$ and was observed in the clinical setting by Ito and colleagues, ${ }^{3}$ using myocardial contrast echocardiography (MCE) with intracoronary injection of sonicated microbubbles.

No reflow or low reflow can be determined by several mechanisms. After thrombolysis, residual stenosis of the infarct related vessel is frequently severe and can be responsible for partial or complete reocclusion or for repetitive episodes of ischaemia and post-ischaemic myocardial and microvascular stunning. ${ }^{4}$ Primary PCI may be accompanied by distal embolisation of platelet aggregates, neutrophil accumulation, and capillary plugging inducing progressive microvascular obstruction despite initial reperfusion. ${ }^{5}$ In other patients, restoration of the blood supply leads to reactive hyperaemia, but also possibly to postreperfusion injury by oxygen-free radicals, inducing cellular oedema. After optimisation of vessel patency by successful stent placement, early and sustained improvement of coronary flow reserve is usually observed, consistent with gradual resolution of post-ischaemic vascular stunning. ${ }^{6}$ In other words, after early recanalisation, myocardial perfusion can dynamically evolve in two opposite directions: complete or at least partial recovery of microvascular integrity, or progressively irreversible microvascular damage, frequently accompanied by intramyocardial haemorrhage. ${ }^{7}$
Department of Cardiology, Ipierard@chu.ulg.ac.be B - 4000 Liege, Belgium;

\section{SALVAGE VERSUS NECROSIS}

The transmural extent of myocardial and microvascular necrosis is highly variable from patient to patient. The major determinants are presence and adequacy of collateral circulation, time to treatment, and therapeutic efficacy. The time from symptom onset to the initiation of treatment is the sum of three individual periods of time: patient's delay in seeking medical attention, transport time, and door-toneedle or door-to-balloon time. Patients should be educated to react early, the thrombolytic agent or the adjunctive treatment as a preparation for PCI can be given during transport, and door-toneedle or door-to-balloon times need to be improved.

\section{IMMEDIATE ASSESSMENT}

How is the efficacy of the initial treatment assessed? There is no simple answer to this question. The integration of several types of information is required as usual in medicine. Simple, non-invasive, widely available, and easily repeatable tools are preferable. They should be applied timely, when it is necessary to make a medical decision. First, chronologically, it is important to appropriately select patients in whom rescue PCI is indicated after failed thrombolysis. Persistence of pain and absent or insufficient ST segment resolution can be useful. The precise time and cut-off points of ST segment resolution remain, however, unclear. MCE can assess the absence or presence of microvascular perfusion, a prerequisite of tissue viability. This can be done rapidly, at the bedside, by intravenous bolus injection and/or continuous infusion of a contrast agent consisting of small microbubbles passing first through the pulmonary capillaries, and then opacifying the left ventricular cavity and enhancing the myocardium. MCE detects contrast microbubbles at the capillary level and allows assessment of myocardial blood volume and red cell velocity. Several modalities are available, mainly high power triggered imaging or low power continuous imaging after a flash echo at high mechanical index in order to destroy all microbubbles and then observe microbubble replenishment, indicating refilling of myocardial capillaries. For example, fig 1 illustrates the usefulness of early MCE at the end of thrombolytic infusion. The left panel shows an end systolic triggered image in the four chamber view. The left ventricle, septum, and apex are opacified. The lateral wall is thin, as a result of akinesis, and does not show contrast enhancement. Coronary angiography was immediately performed after MCE in this patient: the circumflex artery was occluded. 

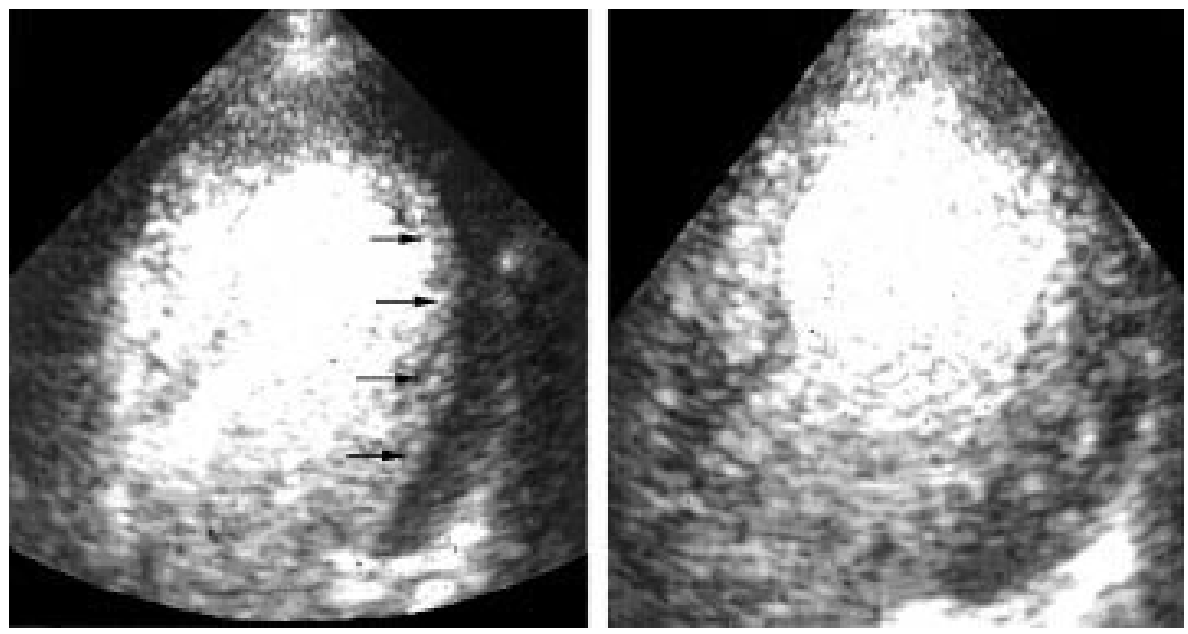

Figure 1 End systolic triggered images in the apical four chamber view after intravenous contrast administration in a patient with acute lateral myocardial infarction. Left: No reflow at the end of thrombolytic infusion. Rescue percutaneous coronary intervention was decided. Right: Before hospital discharge-contrast enhancement of the affected area and thickness similar to the remote regions, demonstrating both microvascular and contractile recovery.
Rescue PCI was successful. The right panel shows a similar image before hospital discharge. The lateral wall is well opacified and its end systolic thickness is similar to that of the remote regions, indicating both microvascular and contractile recovery.

Similarly, MCE can be performed immediately after primary PCI to assess the absence or presence and extent of microvascular salvage. Early identification of patients with extensive no reflow would indeed be particularly useful if novel pharmacological adjunctive agents may enhance more optimal reperfusion. A potential pitfall of immediate MCE study is the frequent occurrence of post reflow hyperaemia of the risk area, which may significantly underestimate the degree of necrosis. However, even if perfusion remains preserved in necrotic regions early after restoration of patency, microvascular reserve is impaired. Vasodilatation by adenosine administration can unmask abnormalities in flow reserve. MCE in conjunction with a coronary vasodilator predicts infarct size more accurately, early after recanalisation in experimental studies. ${ }^{8}$ Other methods can be used to determine infarct size, but are less widely available or cannot be performed at the bedside. Magnetic resonance imaging (MRI) has been shown, in both experimental and clinical studies, to precisely measure infarct size with necrosis-avid MRI contrast agents.

\section{PERFUSION AND FUNCTION AFTER THE ACUTE PHASE: SPATIAL HETEROGENEITY}

After the immediate therapeutic decisions have been reached, it is important to stratify the patient's risk by differentiating between necrotic tissue and viable myocardium. A transmural infarction is indeed an important determinant of adverse clinical outcome, in relation to myocardial rupture, infarct expansion, and ventricular remodelling. Myocardial salvage is increasingly obtained in the present era of early intervention. After thrombolytic treatment, viable regions may be stunned or hibernating. The myocardial state is mainly determined by the level of flow and flow reserve, but there is usually an admixture of necrotic and viable myocytes in a highly variable proportion from patient to patient. This spatial heterogeneity also concerns the microvasculature, with variable regions of no reflow, low reflow, reduced flow reserve or normalised flow. When necrosis predominates and viability is limited to a small epicardial ring, infarct expansion and ventricular remodelling may be prevented, even if contractile recovery will not occur, since the endocardial half of the ventricular wall mainly contributes to myocardial thickening at rest. ${ }^{10}$

Numerous techniques are potentially useful in the detection of viable myocardium. As viability depends on preserved perfusion and cell integrity, nuclear methods have often been used, such as thallium or technetium-99m sestamibi single photon emission tomography. MIBI has the potential advantage of delineating the risk area if an injection was made before the acute therapeutic intervention. A second study can quantify the extent of perfusion recovery. The main limitation of nuclear techniques, including the less available positron emission tomographic imaging, is a limited spatial resolution precluding distinction between the epicardial and endocardial layers. Conventional echocardiography at rest cannot distinguish between akinetic necrotic or viable myocardium because of the threshold phenomenon, but hypokinesis in the first hours or days implies necrosis to $<20 \%$ of the inner endocardium. With its high versatility, echocardiography is an attractive tool, as several ultrasonic modalities can provide complementary information. MCE can evaluate the microvasculature. Doppler tissue imaging can quantitate myocardial velocity and deformation patterns, enabling normal, ischaemic or stunned myocardium to be characterised. ${ }^{11}$

Echocardiography has high spatial and temporal resolutions. Both MCE and tissue Doppler modalities have the potential to provide specific information on the different myocardial layers.

\section{PERFUSION AND FUNCTION: TEMPORAL HETEROGENEITY}

Recovery in contraction after acute myocardial infarction implies a sufficient proportion of viable myocardium with good flow reserve. Functional recovery can be predicted by the recruitment of contractile reserve during dobutamine echocardiography. Sustained improvement is a marker of stunned myocardium; a biphasic response suggests limited flow reserve, usually because of a severe residual stenosis.

The presence of myocardial perfusion with MCE, a prerequisite for myocardial viability, can also predict recovery in regional and global function and does not require an inotropic challenge. However, serial studies may be necessary. Indeed, post-ischaemic myocardial perfusion has a dynamic nature. Microvascular damage, as assessed shortly after reflow, may be reversible even in an area of no reflow. ${ }^{12}$ In this issue of Heart, Galiuto and colleagues ${ }^{13}$ demonstrate that microvascular reflow can improve even after the initial 24 hours in some patients, and that this improvement correlates with improved left ventricular function and volumes. These observations indicate that the window for salvage may exceed the first 24 hours, at least in some patients, and suggest that new medical therapeutic interventions need to be tested. 


\section{CONCLUSION}

Spatial and temporal evolution of regional perfusion and function are highly variable after acute myocardial infarction. Assessment at a single point in time is not appropriate. Serial evaluation is usually required. Echocardiography, with its different modalities, appears ideal as it has the potential for evaluating both perfusion and function, in the different layers, at baseline and throughout administration of a vasodilator or an inotropic agent.

\section{REFERENCES}

1 Kloner RA, Rude RE, Carlson N, et al. Ultrastructural evidence of microvascular damage and myocardial cell injury after coronary artery occlusion: which comes first? Circulation 1980:62:945-52.

2 Kloner RA, Ganote CE, Jennings RB. The "no reflow" phenomenon after temporary coronary occlusion in the dog. J Clin Invest 1974:54:1496-508

3 Ito $\mathbf{H}$, Tomooka T, Sakai N, Yu H, et al. Lack of myocardial perfusion immediately after successful thrombolysis: a predictor of poor recovery of left ventricular function in anterior myocardial infarction. Circulation 1992;85: 1699-705

4 Bolli R, Triana F, Jeroudi MO. Prolonged impairment of coronary vasodilation after reversible ischemia. Evidence for microvascular "stunning". Circ Research 1990;67:332-43.

5 Ambrosio G, Weisman HF, Mannisi JA, et al. Progressive impairment of regional myocardial perfusion after initial restoration of postischemic blood flow. Circulation 1989;80:1846-61
6 Neumann FJ, Kosa I, Dickfeld T, et al. Recovery of myocardial perfusion in acute myocardial infarction after successful balloon angioplasty and stent placement in the infarct-related coronary artery. J Am Coll Cardiol 1997; 30: 1270-6.

7 Asanuma T, Tanabe K, Ochiai K, et al. Relationship between progressive microvascular damage and intramyocardial hemorrhage in patients with reperfused anterior myocardial infarction. Myocardial contrast echocardiographic study. Circulation 1997;96:448-53.

8 Villanueva FS, Camarano G, Ismail S, et al. Coronary reserve abnormalities in the infarcted myocardium. Assessment of myocardial viability immediately versus late after reflow by contrast echocardiography. Circulation 1996;94:748-54.

9 Pislaru SV, Ni Y, Pislaru C, et al. Noninvasive measurements of infarct size after thrombolysis with a necrosis-avid MRI contrast agent. Circulation 1999:99:690-6.

10 Myers JH, Stirling MC, Choy M, et al. Direct measurement of inner and outer wall thickening dynamics with epicardial echocardiography. Circulation 1986;74:164-72.

11 Derumeaux GA, Ovize M, Loufoua J, et al. Assessment of nonuniformity of transmural myocardial velocities by color-coded tissue Doppler imaging: characterization of normal, ischemic, and stunned myocardium. Circulation 2000;101:1390-5.

12 Ito $\mathbf{H}$, Iwakura $\mathrm{K}, \mathrm{Oh} \mathrm{H}$, et al. Temporal changes in myocardial perfusion patterns in patients with reperfused anterior wall myocardial infarction. Circulation 1995;91:656-62.

13 Galiuto L, Lombardo A, Maseri A, et al. Temporal evolution and functional outcome of no reflow: sustained and spontaneously reversible patterns following successful coronary recanalisation. Heart 2003;89:731-7.

\section{IMAGES IN CARDIOLOGY}

\section{Area ablation of ventricular tachycardia in a patient with arrhythmogenic right ventricular cardiomyopathy}

A 26 year old male patient with arrhythmogenic right ventricular cardiomyopathy (ARVC) was referred for catheter ablation of drug refractory ventricular tachycardia (VT). A 12 lead ECG of the VT (CL 190 ms, intermediate axis, left bundle branch block morphology) suggested a right ventricular outflow tract origin (RVOT).

The ablation procedure was guided by a three dimensional Real-time Positioning Management System (RPM). This system uses sonomicrometry to determine the three dimensional location of an ablation catheter relative to two reference catheters positioned into the right atrium and right ventricle. At the onset of the mapping procedure, the right ventricle was depicted as a spherical body. After induction of VT, this spherical body was adjusted to the shape and size of the RVOT by dragging the ablation catheter over the endocardium of the RVOT (below left). Simultaneously, electrograms were recorded sequentially at multiple sites and the local activation time (relative to the

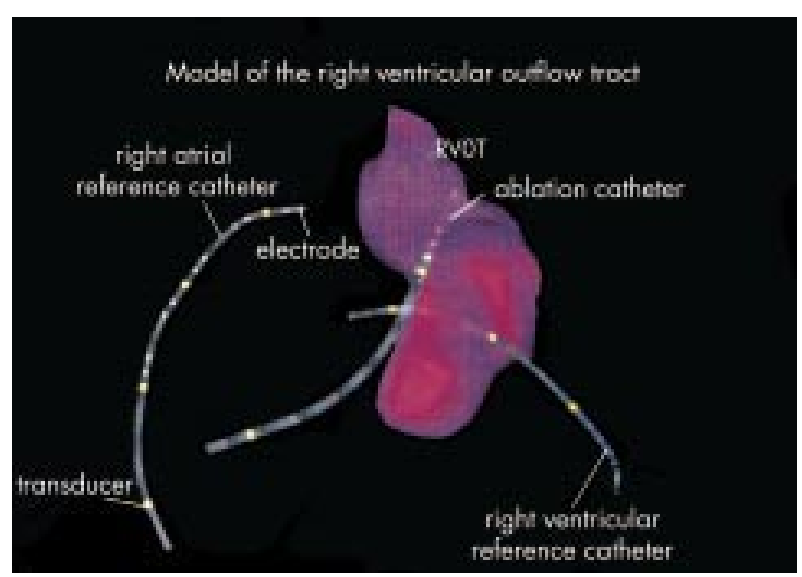

surface ECG) was marked automatically. In this way, a colour coded activation map was superimposed on the RVOT model (below right). At the site of earliest endocardial activity (anterior part of the RVOT), a small area of fragmented electrograms $(2 \times$ $3 \mathrm{~cm}^{2}$ ) was found. The three dimensional endocardial position of this area could accurately be delineated using the RVOT model (white dots). This entire area was ablated. Ablation of this localised area of fragmented electrograms resulted in termination and non-inducibility of the VT after ablation.

This presentation demonstrates facilitation of ablation of VT in a patient with ARVC by accurate delineation of the arrhythmogenic substrate thereby using a three dimensional mapping technique.

N M S de Groot M J Schalii

E E van der Wall m.j.schalij@lumc.nl

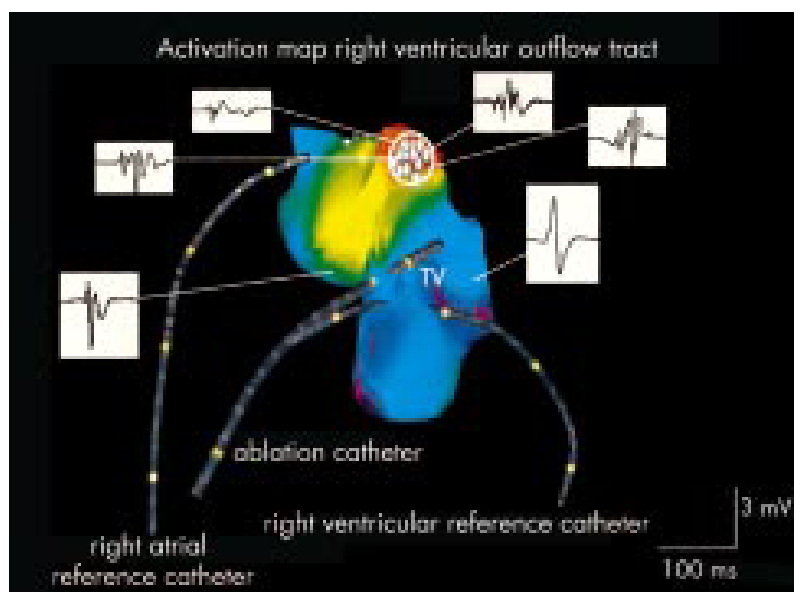

